\title{
Viscosity overshoot in the start-up of uniaxial elongation of low density polyethylene melts
}

Rasmussen, Henrik K.; Nielsen, Jens Kromann; Bach, Anders; Hassager, Ole

Published in:

Journal of Rheology

Link to article, DOI:

$10.1122 / 1.1849188$

Publication date:

2005

Document Version

Publisher's PDF, also known as Version of record

Link back to DTU Orbit

Citation (APA):

Rasmussen, H. K., Nielsen, J. K., Bach, A., \& Hassager, O. (2005). Viscosity overshoot in the start-up of uniaxial elongation of low density polyethylene melts. Journal of Rheology, 49(2), 369-381.

https://doi.org/10.1122/1.1849188

\section{General rights}

Copyright and moral rights for the publications made accessible in the public portal are retained by the authors and/or other copyright owners and it is a condition of accessing publications that users recognise and abide by the legal requirements associated with these rights.

- Users may download and print one copy of any publication from the public portal for the purpose of private study or research.

- You may not further distribute the material or use it for any profit-making activity or commercial gain

- You may freely distribute the URL identifying the publication in the public portal

If you believe that this document breaches copyright please contact us providing details, and we will remove access to the work immediately and investigate your claim. 


\title{
Viscosity overshoot in the start-up of uniaxial elongation of low density polyethylene melts
}

\author{
Henrik Koblitz Rasmussen \\ The Danish Polymer Centre, Department of Manufacturing Engineering and \\ Management, Technical University of Denmark, DK-2800 Kgs. Lyngby, Denmark \\ Jens Kromann Nielsen, Anders Bach, and Ole Hassager ${ }^{\text {a) }}$ \\ The Danish Polymer Centre, Department of Chemical Engineering, Technical \\ University of Denmark, DK-2800 Kgs. Lyngby, Denmark
}

(Received 4 May 2004; final revision received 12 November 2004)

\begin{abstract}
Synopsis
The transient uniaxial elongational viscosity of BASF Lupolen 1840D and 3020D melts has been measured on a filament stretch rheometer up to Hencky strains of 6-7. The elongational viscosity of both melts was measured at $130{ }^{\circ} \mathrm{C}$ within a broad range of elongational rates. At high elongation rates, an overshoot or maximum in the transient elongational viscosity followed by a steady viscosity was observed. The steady elongation viscosity was about $40 \%-50 \%$ less than the maximum at high strain rates. The steady elongational viscosity as a function of the elongation rate, $\dot{\epsilon}$, decreases approximately as $\dot{\epsilon}^{-0.6}$ in both melts at high strain rates. The transient elongational viscosity, measured at a specific elongation rate at $170{ }^{\circ} \mathrm{C}$ on the BASF Lupolen 3020D melt, did not follow the time temperature superposition principle based on linear viscoelasticity during the decrease in the transient elongational viscosity towards the steady state. (C) 2005 The Society of Rheology. [DOI: 10.1122/1.1849188]
\end{abstract}

\section{INTRODUCTION}

About 40 years ago interest in the measurement of elongational viscosity of polymer melts started to grow [Tanner et al. (1998)]. Measurements are most frequently reported either as steady elongational viscosity as a function of elongational rate, or as transient elongational viscosity as function of time for a fixed elongational rate. Generally, it seems to be assumed that the transient elongational viscosity (as opposed to the transient shear viscosity) is an monotone increasing function of time. Raible et al. (1979) presented the first measurements concerning the possible existence of an overshoot in the transient uniaxial elongational viscosity of a polymer melt for a low density polyethylene (LDPE). Contemporary and more recent measurements of elongational viscosity of LDPE [Laun and Münstedt (1978); Münstedt and Laun (1979); Wagner et al. (2000); Bach et al. (2003b)] do not indicate the existence of a maximum in the transient elongational viscosity. The experimental observation of a maximum in the transient elongational viscosity would have important implications for the constitutive modelling of polymer melts.

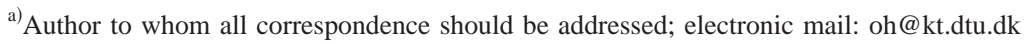


TABLE I. Characterization of the BASF Lupolen 1840D and 3020D polymer melts. Linear viscoelastic spectrum from Bastian (2001) for the Lupolen 3020D.

\begin{tabular}{ccccc}
\hline \hline Polymer melt & LDPE & & LDPE & \\
\hline Product & Lupolen 3020D & & Lupolen 1840D & \\
Producer & BASF & & BASF & \\
$M_{W}(\mathrm{~g} / \mathrm{mol})$ & 300000 & & 490000 & \\
$M_{n}(\mathrm{~g} / \mathrm{mol})$ & 37500 & & 16000 & \\
$M_{W} / M_{n}$ & 8 & & 30.6 & \\
$T_{m}\left({ }^{\circ} \mathrm{C}\right)$ & 114 & & 106 & \\
$T\left({ }^{\circ} \mathrm{C}\right)$ & 170 & $\tau_{i}(\mathrm{~s})$ & 130 & $\tau_{i}(\mathrm{~s})$ \\
Relaxation & $g_{i}(\mathrm{~Pa})$ & $3.278 \times 10^{2}$ & $8.31 \times 10^{2}$ & $8.23 \times 10^{2}$ \\
spectrum at the & $1.117 \times 10^{2}$ & $5.141 \times 10^{1}$ & $2.90 \times 10^{3}$ & $9.55 \times 10^{1}$ \\
temperature $T$ & $1.081 \times 10^{3}$ & $9.052 \times 10^{0}$ & $6.20 \times 10^{3}$ & $1.73 \times 10^{1}$ \\
& $3.904 \times 10^{3}$ & $1.626 \times 10^{0}$ & $1.27 \times 10^{4}$ & $3.35 \times 10^{0}$ \\
& $1.008 \times 10^{4}$ & $2.855 \times 10^{-1}$ & $2.42 \times 10^{4}$ & $5.60 \times 10^{-1}$ \\
& $2.220 \times 10^{4}$ & $4.997 \times 10^{-2}$ & $4.36 \times 10^{4}$ & $1.07 \times 10^{-1}$ \\
& $3.686 \times 10^{4}$ & $9.580 \times 10^{-3}$ & $1.29 \times 10^{5}$ & $1.15 \times 10^{-2}$ \\
& $5.886 \times 10^{4}$ & $1.472 \times 10^{-3}$ & & \\
\hline \hline
\end{tabular}

Recent suggested constitutive equations for branched polymer melts, the Pom-Pom model [McLeish and Larson (1998)] and the molecular stress function (MSF) model [Wagner et al. (2001)], find a monotone increase in the transient elongational viscosity. However, some of the previously suggested constitutive equations were able to predict an overshoot followed by a steady viscosity [see for instance Wagner et al. (1979)]. Hence, it is important to examine the question of whether or not a constitutive equation for branched polymer melts should be able to predict a maximum (followed by a steady state) or a monotone increase in the transient elongational viscosity to the steady viscosity.

We have measured the transient uniaxial elongational viscosity of two BASF LDPE melts, BASF Lupolen 1840D and 3020D using the filament stretch rheometer (FSR). A steady-state viscosity was kept for 1-2.5 Hencky strain units in all measurements.

\section{MATERIAL}

The Lupolen 3020D LDPE melt used here has previously been characterized in shear and uniaxial elongation. Bastian (2001) measured the transient uniaxial elongation viscosities at $170{ }^{\circ} \mathrm{C}$ using the Rheometrics melt extensiometer (RME). Details of the RME rheometer design and experimental procedure are given in Bastian (2001). In this work, uniaxial elongation measurements were performed at $130{ }^{\circ} \mathrm{C}$ for the Lupolen $1840 \mathrm{D}$ and at 130 and $170{ }^{\circ} \mathrm{C}$ for the Lupolen 3020D LDPE melt. Note that our measurements on the Lupolen 3020D are on the same grade as Bastian (2001), but not the same batch. Still, our small-amplitude oscillatory shear measurements (using a plate-plate geometry on a TA Instruments, AR2000) are in agreement with Bastian (2001), and the elongation measurements are in reasonable agreement with Bastian (2001). The properties of the melts are listed in Table I. The linear viscoelastic memory function, $M(s)$, and relaxation modulus, $G(s)$, are described as a sum of exponential function. Thus 


$$
M(s)=\sum_{i}^{N} \frac{g_{i}}{\tau_{i}} \exp \left(-s / \tau_{i}\right)
$$

and

$$
G(s)=\sum_{i}^{N} g_{i} \exp \left(-s / \tau_{i}\right),
$$

where the moduli $g_{i}$ and relaxation times $\tau_{i}$ are given in Table I.

The moduli and relaxation times for the Lupolen 3020D melt [from Bastian (2001)] are listed at $170{ }^{\circ} \mathrm{C}$ in Table I. As our elongation measurements are mainly performed at $130{ }^{\circ} \mathrm{C}$, the linear viscoelastic time-temperature shift factor, $a_{T}$, was measured from 130 to $170{ }^{\circ} \mathrm{C}$. This value, $a_{T}=6.4$, was calculated as the ratio between the angular frequencies, $\omega$, at the cross over point for the two temperatures. The crossover point is defined as the angular frequency where the storage modulus is equal to the loss modulus; $G^{\prime}(\omega)=G^{\prime \prime}(\omega)$. The storage modulus and loss modulus were measured in small amplitude oscillatory shear flow using a plate-plate geometry on an AR2000 rheometer from TA Instruments. The steady shear viscosity was also measured using the same rheometer in a cone-plate geometry (radius $10 \mathrm{~mm}$ and a $1^{\circ}$ cone angle).

We have not been able to measure the largest relaxation times, and consequently the zero-shear viscosity, for the Lupolen $1840 \mathrm{D}$ melt as the linear viscoelastic measurements are affected by crosslinking at the time needed to characterize at angular frequencies lower than $5 \times 10^{-4} \mathrm{~s}^{-1}$ at $130{ }^{\circ} \mathrm{C}$. The molecular structure of the Lupolen $1840 \mathrm{D}$, among other LDPE melts, has been discussed in Nordmaier et al. (1990a, 1990b).

\section{THE FILAMENT STRETCHING RHEOMETER}

McKinley and Sridhar (2002) have given an extensive review of elongational rheometry with particular emphasis on the filament stretch rheometer. The apparatus used in our experiments is a vertical filament stretching rheometer, surrounded by a thermostated environment, where the bottom plate is stationary and the upper plate is pulled. Basically a cylindrical sample is placed between two parallel plates and at times, $t \geqslant 0$, the upper plate is pulled decreasing the midradius in the sample, $R(t)$, exponentially in time. This applies a constant elongation rate, $\dot{\epsilon}_{0}$, at the centre of the sample from $t=0$. At $t<0$ the strain rate is 0 . A laser is used to measure the mid-diameter of the filament during an experiment. The average strain rate, $\dot{\epsilon}_{0}$, is calculated from the measurement of the diameter by fitting an exponential function $R(t)=R_{0} \exp \left(-\dot{\epsilon}_{0} t / 2\right)$ where $R_{0}$ is the initial sample radius and $R(t)$ the actual sample radius. Generally, the relative deviation between measurements and fit never exceeded $4 \%$. At elongation rates lower than $0.1 \mathrm{~s}^{-1}$ the deviation between measurements and fit was never above $2 \%$ and usually within $1 \%$.

The advantage of the FSR compared to conventional techniques for elongation rheometry is the ability to predict the location of the symmetry plane (the necking) of the sample. Subsequently this critical region can be monitored, and the distance between the end plates adjusted online obtaining a constant stretch rate at the neck. Notice that the laser simultaneous measures the diameter of the filament and records video images of the area surrounding the place where the diameter is measured, giving a good assessment of the local sample deformation as a function of strain. Thus it is ensured that the sample stays centered (symmetric necking) and the radius of curvature of the neck in the axial direction is sufficiently large. 


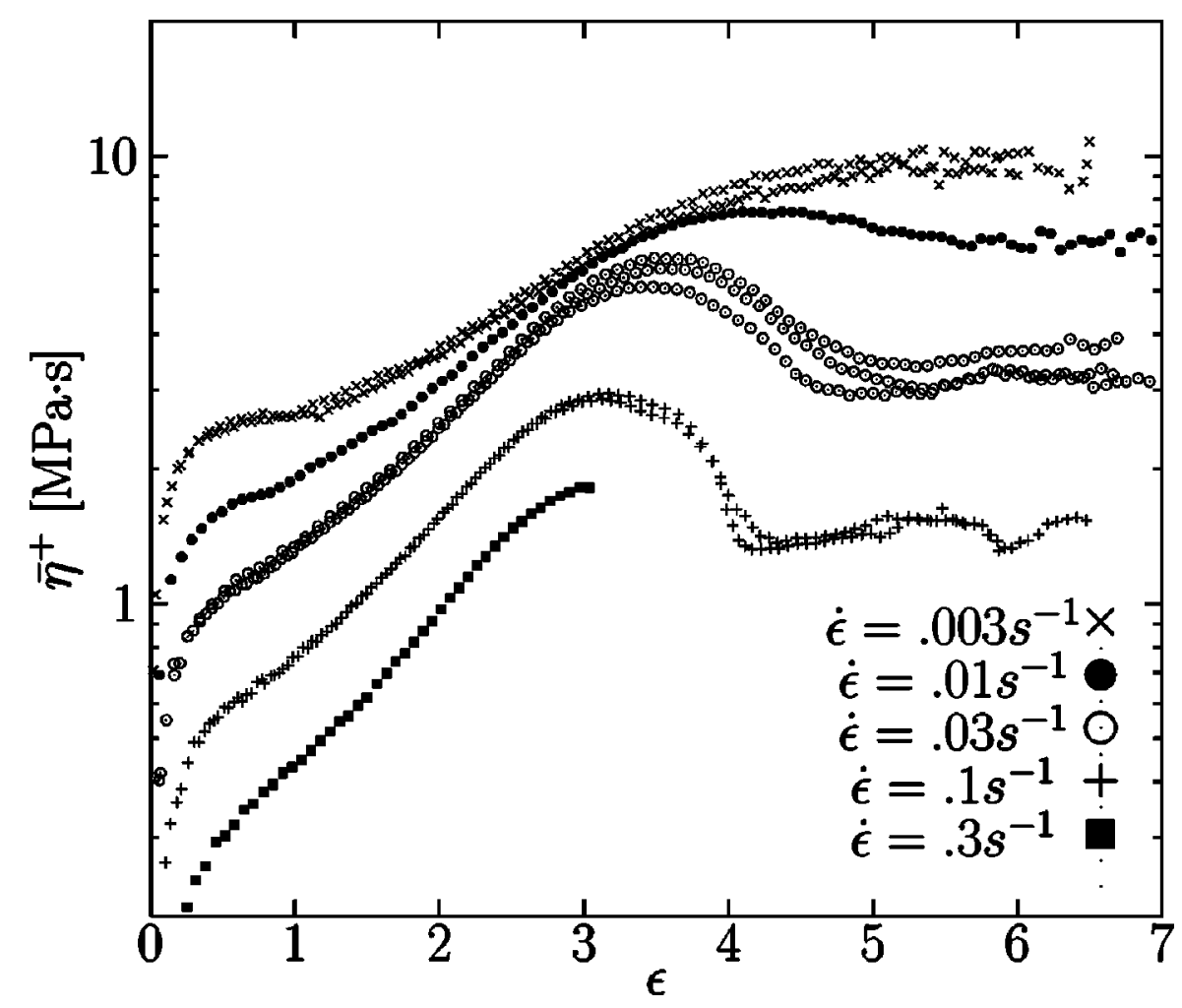

FIG. 1. The uncorrected transient elongation viscosities $\bar{\eta}^{+}$of Lupolen $3020 \mathrm{D}$ measured at $130{ }^{\circ} \mathrm{C}$, using Eq. (3), shown as a function of the Hencky strain, $\epsilon . \bar{\eta}^{+}$are measured at five different elongational rates $\dot{\epsilon}$ as shown in the figure.

Recently [Bach et al. (2003a)] the FSR rheometer has been improved using a closed loop proportional regulator scheme to control the deformation of the sample. This replaces the iterative Orr-Sridhar method [Bach et al. (2003b)] and has allowed measurements at considerably higher strain values than presented before.

During an experiment both the sample radius $R(t)$ and the axial force $F(t)$ in the filament were measured as a function of time. The axial force is measured on the fixed bottom end plate, which is mounted on a weight cell placed outside the thermostated environment.

When the average strain rate had been established, the transient elongational viscosity $\bar{\eta}^{+}(t)$ was calculated using

$$
\bar{\eta}^{+}(t)=\frac{F(t)-m_{1} g}{\pi R(t)^{2} \dot{\epsilon}_{0}},
$$

where the measured force, $F$, has been corrected by the weight of lower half of the polymer filament, $m_{1}$ and the gravitational acceleration $g$ [Szabo (1997); Szabo and McKinley (2003)]. This weight is measured by forcing the filament to break in the symmetry plane after the end of an experiment.

This paper presents measurements of transient elongational viscosity of up to a Hencky strains of 7. The Hencky strain is defined as $\epsilon(t)=-2 \ln \left[R(t) / R_{0}\right]$ where $R_{0}$ is the initial sample radius and $R(t)$ the actual sample radius, measured at the symmetry plane of the filament as a function of time. 


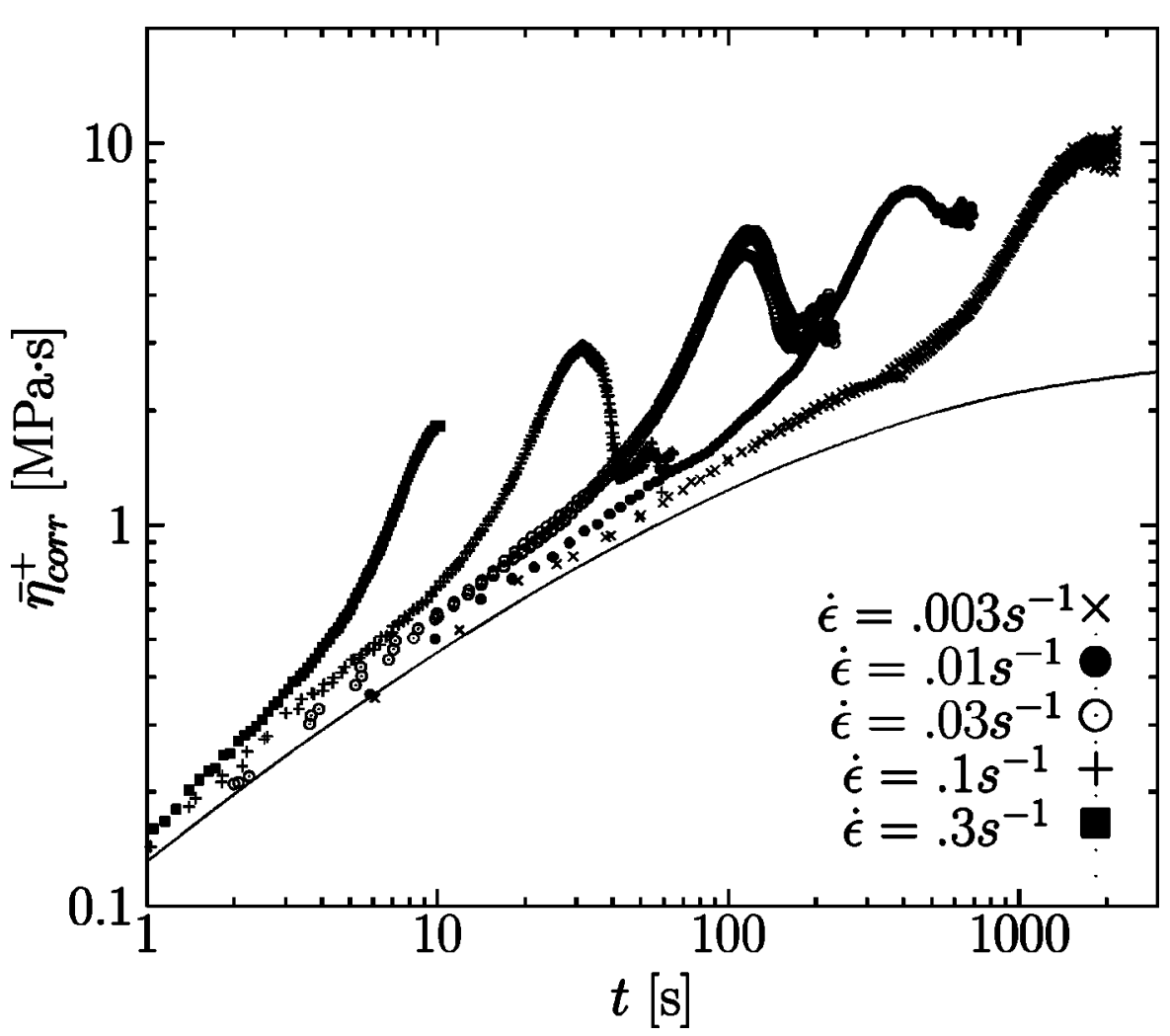

FIG. 2. The corrected transient elongation viscosities $\bar{\eta}^{+}$of Lupolen $3020 \mathrm{D}$ at $130{ }^{\circ} \mathrm{C}$, using Eq. (4), shown as a function of the time, $t$. The measurements are the same as in Fig. 1. The line is the linear viscoelastic prediction of the transient elongational viscosity.

\section{LDPE TEST SAMPLE}

The two LDPE polymers, supplied in pellets, were pressed into cylindrical test specimens by a Carver hydraulic press at $130{ }^{\circ} \mathrm{C}$, with radius $R_{0}=4.5 \mathrm{~mm}$ and lengths, $L_{0}$ $=2.5 \mathrm{~mm}$, giving an aspect ratio $\Lambda_{0}=L_{0} / R_{0}=0.56$. A low initial aspect ratio is required for measuring on LDPE melt at high elongation rates in the FSR. At low aspect ratio, the location of the neck in the circular sample, during the extension, is predictably placed in the middle of the filament, creating the symmetry plane, where the laser sheet is located. By contrast, with a high aspect ratio, an unexpected neck located closer to one of the end plates typically occurs. As a consequence, the filament will break asymmetrically and the symmetry plane at the mid filament is lost. The procedure designed to apply the cylindrical sample to the cylindrical shaped stainless steel end plates is described in detail in Bach et al. (2003b).

At small strains the shear components in the deformation field adds an extra force contribution during start-up, especially at small aspect ratios. The extra shear component originates from the no slip condition at the rigid end plates. For Newtonian fluids this reverse squeeze flow problem can be modeled analytically eliminating the effect of the shear by a correction factor [Spiegelberg and McKinley (1996)]:

$$
\bar{\eta}_{\mathrm{corr}}^{+}=\bar{\eta}^{+}\left[1+\frac{\exp (-7 \epsilon / 3)}{3 \Lambda_{0}^{2}}\right]^{-1},
$$

where $\bar{\eta}_{\text {corr }}^{+}$is the corrected transient uniaxial elongation viscosity. 


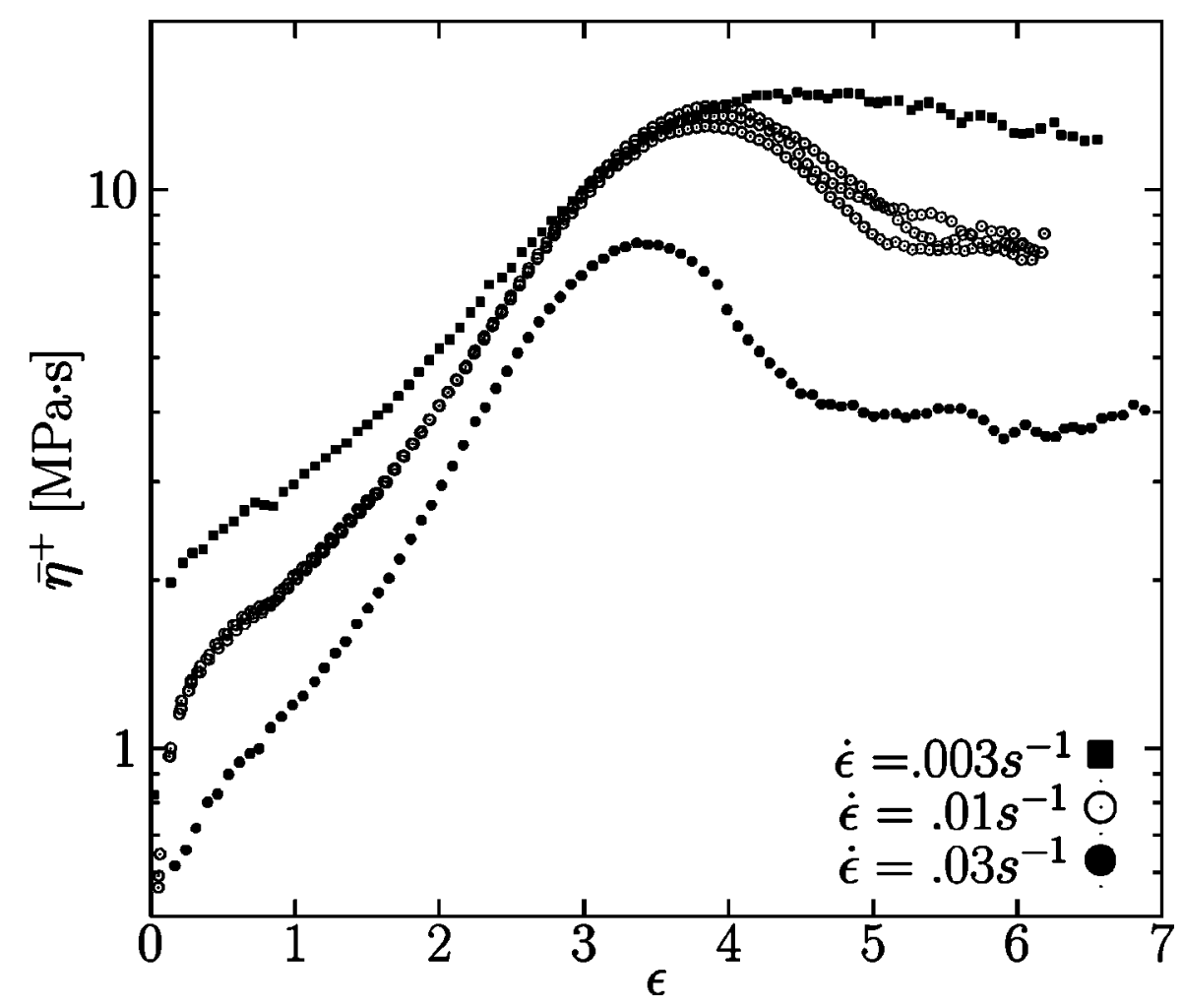

FIG. 3. The uncorrected transient elongation viscosities $\bar{\eta}^{+}$of Lupolen $1840 \mathrm{D}$ measured at $130{ }^{\circ} \mathrm{C}$, using Eq. (3), shown as a function of the Hencky strain, $\epsilon \cdot \bar{\eta}^{+}$are measured at three different elongational rates $\dot{\epsilon}$ as shown in the figure.

This correction is analytically correct for very small strains (e.g., basically $\epsilon=0$ ) for all type of fluids. At increasing strains the corrections are less appropriate, where the effect of the correction fortunately vanishes. In this work we have chosen to present the data both uncorrected and corrected in the presentation of the elongation measurements, as we also prefer to present the raw data. For the aspect ratio used here, this extra force contribution is negligible after about one strain unit. This was also demonstrated theoretically in Kolte et al. (1997) and experimentally in Bach et al. (2003b) for polymer melts.

\section{ELONGATIONAL VISCOSITY MEASUREMENTS}

During extension, the molten polymer filament is surrounded by nitrogen. Hence, there is a lower limit on the extension rate at which sagging of the filament can be neglected during an experiment. A relevant measure of the magnitude of gravitational forces relative to the viscous forces is the ratio of the Reynolds number to the Froude number, $L_{0} \exp (\epsilon) \rho g /\left(2 \dot{\epsilon}_{0} \bar{\eta}^{+}\right)$, where $\rho$ is the density of the polymer melt. From this we estimate that sagging of the polymer melt can be neglected, as this number is less than 0.2 in all performed experiments.

The effect of the surface tension can be neglected as the surface elasticity number [Spiegelberg and McKinley (1996); Rasmussen and Hassager (2001)] do have values of 


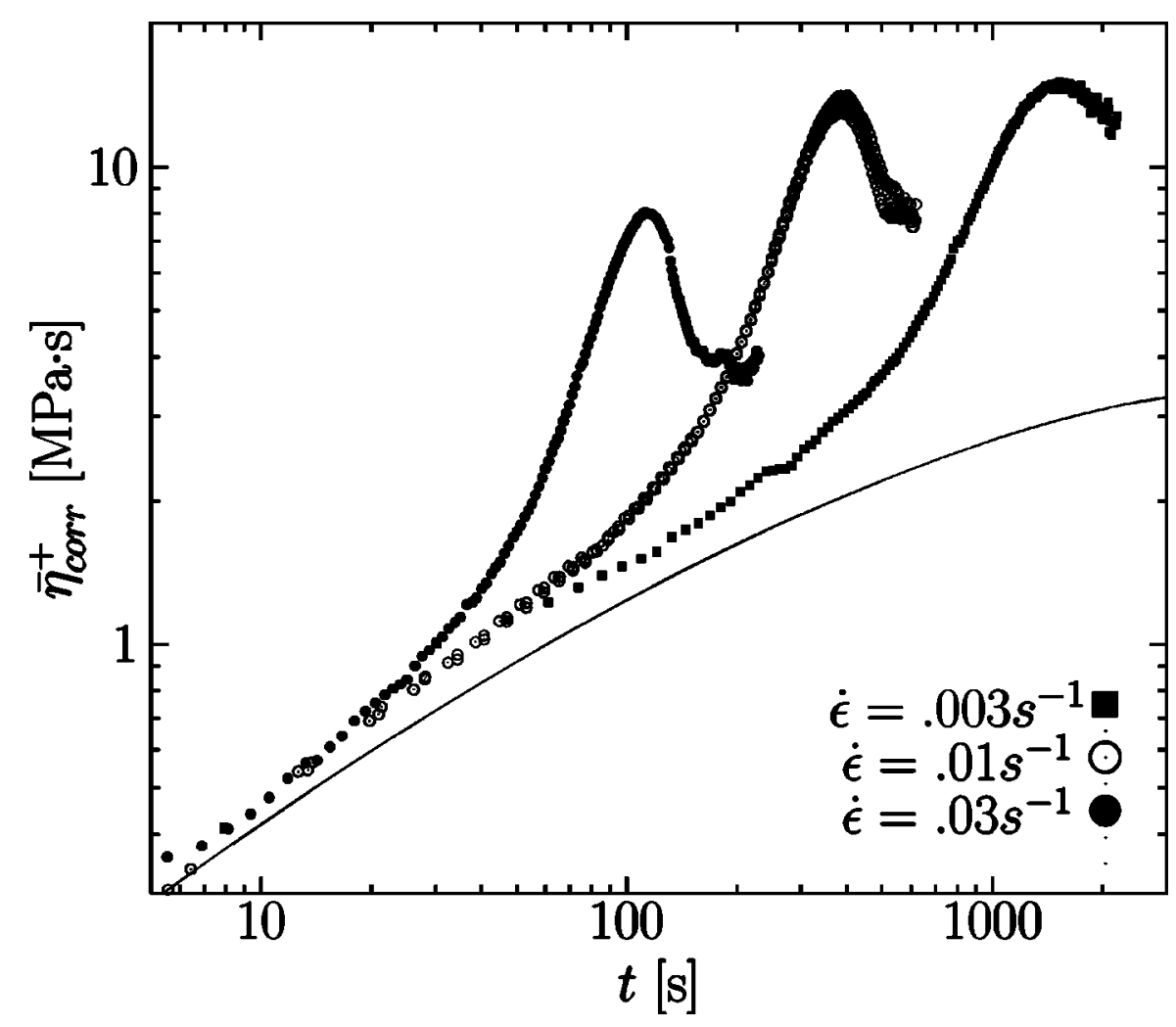

FIG. 4. The corrected transient elongation viscosities $\bar{\eta}^{+}$of Lupolen $1840 \mathrm{D}$ at $130{ }^{\circ} \mathrm{C}$, using Eq. (4), shown as a function of the time, $t$. The measurements are the same as in Fig. 3. The line is the linear viscoelastic prediction of the transient elongational viscosity.

the order $10^{-5}$ for the two polymer melts. The surface elasticity number, $\phi$, is calculated as the ratio of stresses due to surface tension, $\sigma / R$, relative to the elastic modulus as $\phi$ $=\sigma /(R \cdot G)$, where $G$ is the plateau modulus.

We present our complete data series, measured at $130{ }^{\circ} \mathrm{C}$, for the LDPE melts in Figs. 1-4. In Figs. 1 and 3 the uncorrected transient elongation viscosities, using Eq. (3), are shown as a function of the Hencky strain. Keep in mind that our data should not be used without correction for Hencky strains less than unity. In Figs. 2 and 4 these measurements as a function of time are shown corrected, using Eq. (4). Note that the theory of linear viscoelasticity (the line in Figs. 2 and 4) may be used to predict the corrected transient elongational viscosity at (very) small strains from the relaxation modulus. The experiments show good agreement with the predictions from linear viscoelasticity. In order to illustrate the reproducibility some of the measurements have been repeated two or three times. As the strain or elongation rates of the repeated experiments are within $1 \%$, the measurements are not labeled individually. Overall the experiments show differences of about $10 \%-20 \%$.

We notice that the elongational viscosity reaches a steady state in all experiments. The plateau values of elongational viscosity are constant within about $10 \%$ and the steady state has been measured during at least one strain unit for both melts. The only exception is at the highest strain rate. At the highest elongation velocity, it has not been possible to adjust the plate motion to avoid premature sample failure. This is due to the strong 


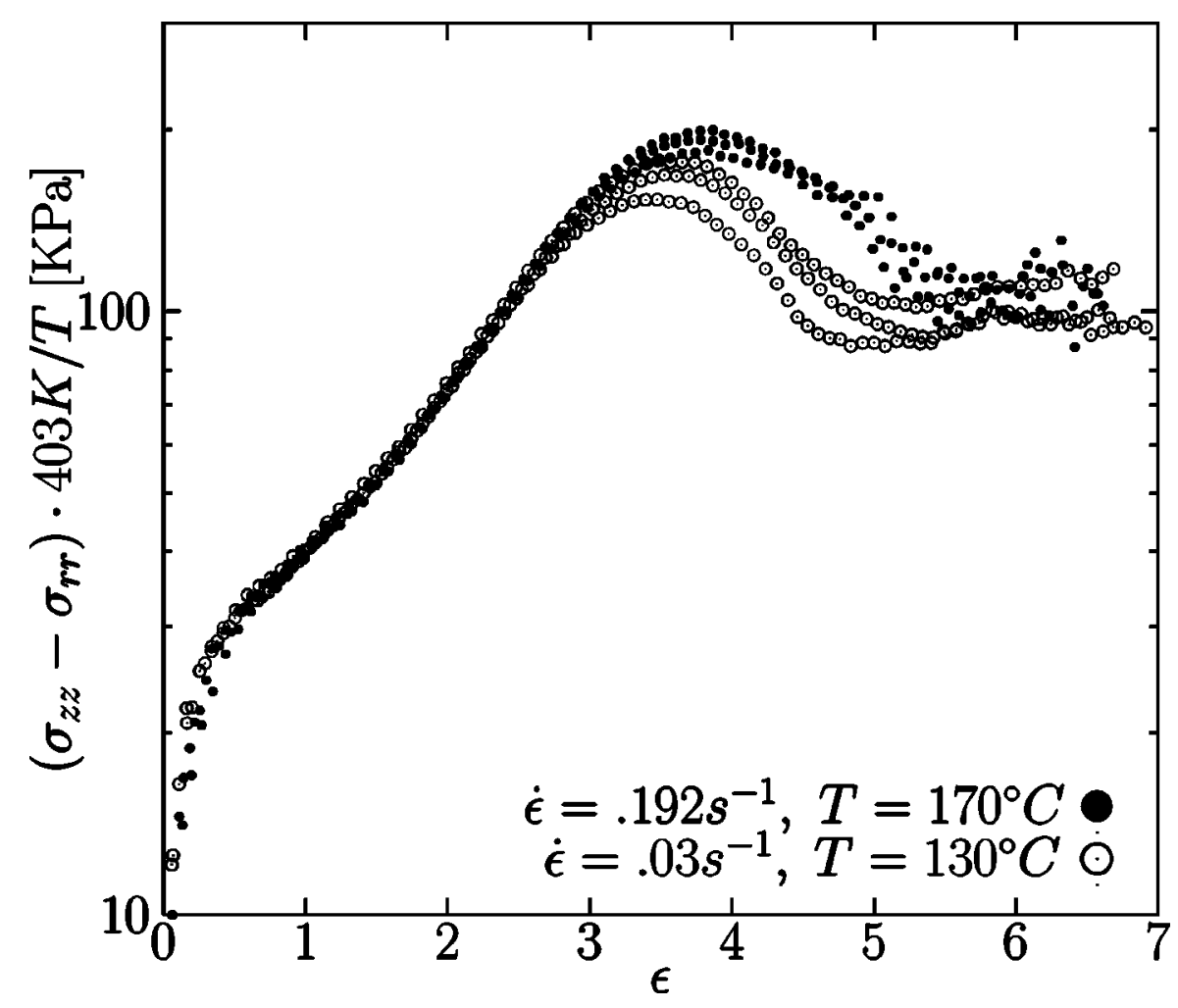

FIG. 5. The uncorrected extensional stress, $\sigma_{z z}-\sigma_{r r}=\bar{\eta}^{+}(t) \epsilon_{0}$, divided with the absolute temperature, $T$, as a function of the Hencky strain, $\epsilon$ of Lupolen 3020D melt. $\sigma_{z z}-\sigma_{r r}$ or $\bar{\eta}^{+}(t)$ are measured at $130{ }^{\circ} \mathrm{C}$ and $\dot{\epsilon}$ $=0.03 \mathrm{~s}^{-1}$ (the measurements are the same as in Fig. 1) and at $170{ }^{\circ} \mathrm{C}$ and $\dot{\epsilon}=0.192 \mathrm{~s}^{-1}$.

nonlinear elastic behavior of the LDPE melts. Note, the small fluctuations in the steadystate values are correlated with small changes in the instantaneous strain rate.

The elongation measurements on the BASF Lupolen 3020D were also performed at the elevated temperature $170{ }^{\circ} \mathrm{C}$ to examine the effects of time-temperature superposition. In Fig. 5 we plot the extensional stress $\left[\sigma_{z z}-\sigma_{r r}=\bar{\eta}^{+}(t) \dot{\epsilon}_{0}\right]$ divided with the absolute temperature as a function of the Hencky strain. $\sigma_{z z}$ and $\sigma_{r r}$ are the axial and radial stress components, respectively. The three measurements at strain rate $0.03 \mathrm{~s}^{-1}$ from Fig. 1 (at $130{ }^{\circ} \mathrm{C}$ ) and the three measurements performed at $170{ }^{\circ} \mathrm{C}$ at an elongation rate of $a_{T} \cdot 0.03 \mathrm{~s}^{-1}=0.192 \mathrm{~s}^{-1}$ are shown.

To test whether or not the elongation measurements at $170{ }^{\circ} \mathrm{C}$ are affected by crosslinking during the thermostating of the LDPE sample, measurements were performed decreasing the time of the thermostating period of about a factor of 2 . All measurements were within the experimental accuracy.

\section{DISCUSSION}

The measurements below elongation rates of $0.3 \mathrm{~s}^{-1}$ reach Hencky strains of 6-7, showing a convincing steady plateau at high strain. This plateau is reached after going through a significant maximum in the transient elongational viscosity at high strain rates. Depending on the elongational rate, the steady elongational viscosities are reduced by factors in the range 1.0-1.9 for both melts. A monotone increase in the transient elon- 


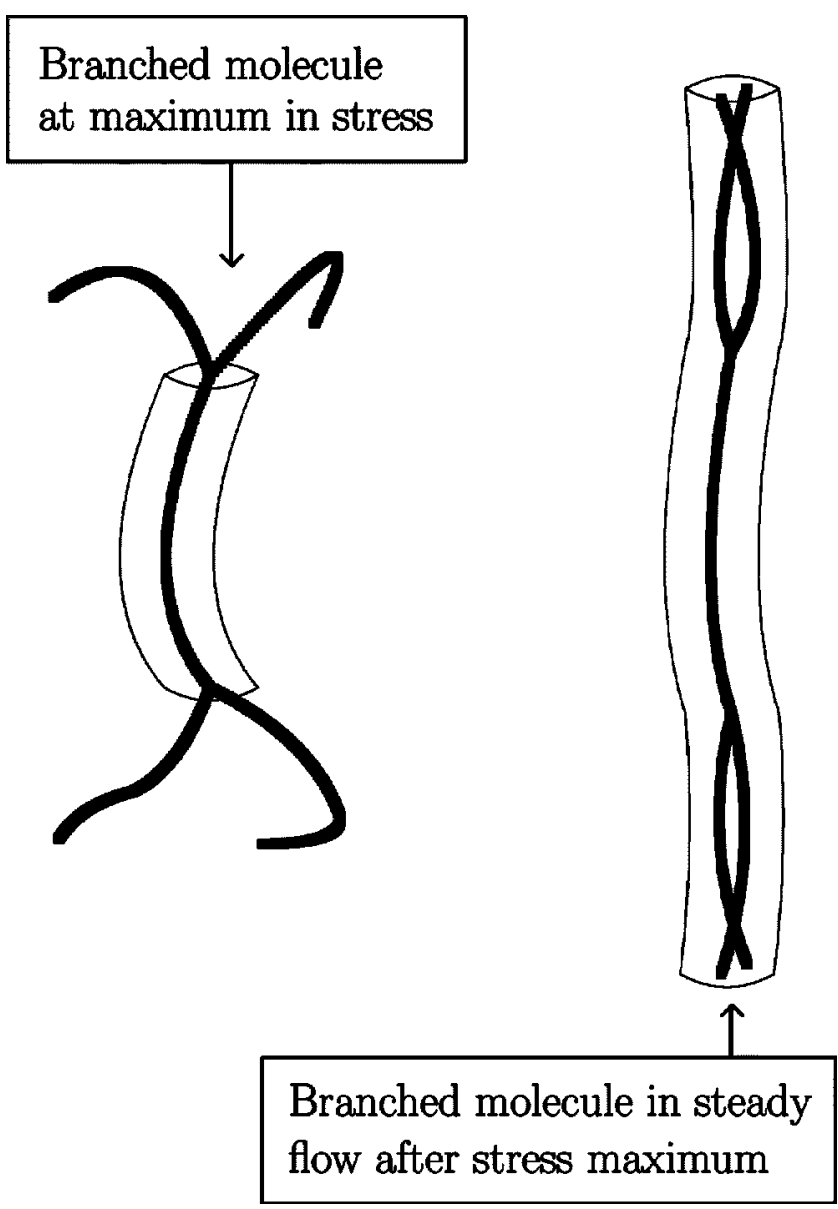

FIG. 6. Interpretation of reduction in stress in terms of Pom-Pom picture. At the maximum in stress, the arms contribute to the tension in the backbone. At steady state, the molecule becomes effectively a linear polymer without arms.

gational viscosity to its steady viscosity is measured at low elongation rates. At high strain rates the maximum can erroneously be interpreted as a steady viscosity, as the transient viscosity does not change more than $10 \%$ within one Hencky strain unit. However, the steady viscosity is reached about one strain unit later than the maximum. We interpret the relatively steep drop in the transient elongational viscosity as a result of a sudden retraction of sidechains from the backbone of the branched polyethylene molecule, into the tube occupied by the backbone. This is illustrated in Fig. 6. Notice that the Pom-Pom model [McLeish and Larson (1998)] does not predict a reduction in the stress, since the authors did not consider a reduction in crossbar tension as a result of arm retraction into the backbone tube. The earlier maximum in the transient elongation viscosity is related only to branched polymer melt. Linear melts do show a monotone increase in the transient elongational viscosity to its steady viscosity [Bach et al. (2003a, 2003b)].

In Fig. 5 we compare measurements performed at $130{ }^{\circ} \mathrm{C}$ with measurements performed at $170{ }^{\circ} \mathrm{C}$, but shifted to $130{ }^{\circ} \mathrm{C}$ by the shift factor obtained in linear viscoelastic measurements. Up to a Hencky strain somewhat below 3, practically all data collapse on 


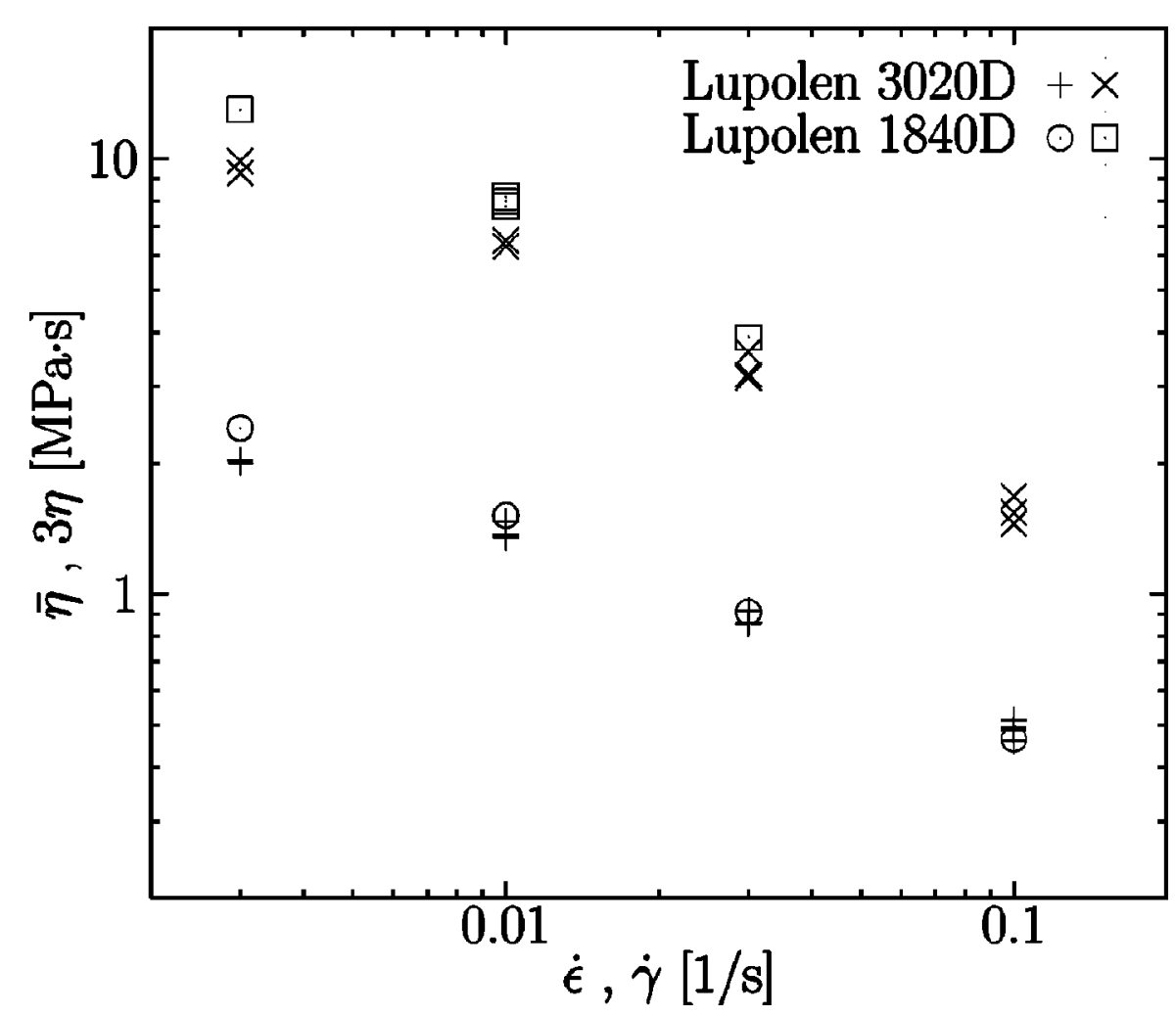

FIG. 7. The steady elongation viscosities, $\bar{\eta}(\times$ and $\square)$, and three times the steady shear viscosities, $3 \eta(+$ and $\circ$ ), of Lupolen 1840D ( $\square$ and $\circ$ ) and Lupolen 3020D $\left(\times\right.$ and + ), measured at $130{ }^{\circ} \mathrm{C}$, shown as a function of the elongation rate, $\dot{\epsilon}$, and shear rate $\dot{\gamma}$, respectively.

a single curve [see also Münstedt and Laun (1979)]. Hence, the measurements follow the time temperature superposition principle based on linear viscoelasticity. Above a Hencky strain value of 3 , the deviations between the measurements at 170 and $130{ }^{\circ} \mathrm{C}$, as presented in the figure, are up until $40 \%$. This is due to the decrease in transient elongational viscosity at $170{ }^{\circ} \mathrm{C}$ happening about one strain unit later than the measurements at $130^{\circ} \mathrm{C}$. As the steady state is reached, all the measurements in Fig. 5 match within the relative large experimental scattering. The delay of the decrease in the transient elongational viscosity emphasize that the time constant in the underlying dynamics, which we expect is the sudden retraction of side chain, do scale differently than the overall dynamics of the polymer.

In Fig. 7 we show the steady shear, $3 \eta$, and elongational viscosity, $\bar{\eta}$, as a function of, respectively, the shear, $\dot{\gamma}$, and elongation rate, $\dot{\epsilon}$, for the Lupolen 1840D and 3020D melt. At high elongation rates the viscosity decreases approximately as $\dot{\epsilon}^{-0.6}$ in both melts. Bach et al. (2003a) and by Gupta et al. (2000) have observed the same slope within the experimental accuracy, measuring steady elongational viscosity on monodisperse (linear) polystyrene melts and solutions, respectively. Bach et al. (2003b) have also observed this phenomenon measuring on linear low density polyethylene (LLDPE) melts.

The observation that the steady elongational viscosity decreases with the same slope as linear melts and follows the time temperature superposition principle indicate that all sidechains in fact retract, leaving only one effective chain expected to behave similar to a linear polymer. We take this as an indication, that the LDPE arms are indeed collapsed 
into the tube occupied by the backbone, concluding that the LDPE in the steady state behaves effectively as a linear polymer. The asymptotic behavior of elongational viscosity for large elongational rates has been analyzed by McKinley and Sridhar (2002). In particular they show that the inclusion of finite extensibility [Wiest (1989)] in the Giesekus anisotropic friction dumbbell model yields an asymptotic relation $\dot{\epsilon}^{-1 / 2}$, which is close to our observations.

Notice that the steady shear viscosity changes with almost the same exponent as the elongational viscosity. The steady elongational viscosity is only about four times higher than (three times) the steady shear viscosity.

\section{ACKNOWLEDGMENTS}

The authors gratefully acknowledge financial support to the Graduate School of Polymer Science from Danish Research Training Council and the Danish Technical Research Council to the Danish Polymer Centre.

\section{APPENDIX}

In this paper we have reported and discussed experimental observations of time dependent uniaxial elongation viscosities of LDPE melts. One of the classical theoretical discussions in the literature is whether or not a constitutive equation for branched polymeric melt should be separable in time and strain. See Wagner et al. (2001) for more details. A constitutive model as the Pom-Pom model is not separable in time and strain whereas the MSF model is. In this appendix we will apply the assumption of time and strain separability on our Lupolen 3020D elongation data as a contribution to the ongoing discussion. In the start-up of an uniaxial elongation flow the time and strain separability requires that the stress or viscosity can be written as [see Wagner (1978)]:

$$
\bar{\eta}^{+}=\frac{G(t) S\left(\dot{\epsilon}_{0} t\right)}{\dot{\epsilon}_{0}}+\frac{1}{\dot{\epsilon}_{0}} \int_{0}^{t} M\left(t-t^{\prime}\right) S\left[\dot{\epsilon}_{0}\left(t-t^{\prime}\right)\right] d t^{\prime}
$$

where $S(\epsilon)$ is a strain dependent function, $t$ the present time, and $G(t)$ and $M\left(t-t^{\prime}\right)$ are the linear viscoelastic relaxation modulus and memory function, respectively. The memory function and relaxation modulus are defined in Eqs. (1) and (2).

Using Eq. (A1) the strain function can be calculated from the viscosity data as

$$
S(\epsilon)=\frac{\bar{\eta}_{\mathrm{corr}}^{+}(\epsilon) \dot{\epsilon}_{0}}{G\left(\epsilon / \dot{\epsilon}_{0}\right)}-\int_{0}^{\epsilon} \bar{\eta}_{\mathrm{corr}}^{+}\left(\epsilon^{\prime}\right) \frac{M\left(\epsilon^{\prime} / \dot{\epsilon}_{0}\right)}{G\left(\epsilon^{\prime} / \dot{\epsilon}_{0}\right)^{2}} d \epsilon^{\prime}
$$

from Wagner (1978).

Note that we use the corrected viscosity from Eq. (4) in this formula. As mentioned before this correction is analytically correct for very small strains (e.g., basically $\epsilon=0$ ) in all types of fluids. The correction is less appropriate at increasing strains, where the effect of the correction fortunately vanishes. The strain function $S(\epsilon)$, based on the assumption of time and strain separability, is written as a function of the Hencky strain in Fig. 8, using the measurements from Fig. 2. The time strain separability turns out to be an excellent assumption up to a Hencky strain value of 3 , where the assumption is no longer valid. This is the same strain as the failing of time-temperature superposition. On the basis of our performed experiment we cannot preclude that the principle of time strain separability may be valid at higher strain rates than shown in Fig. 8. 


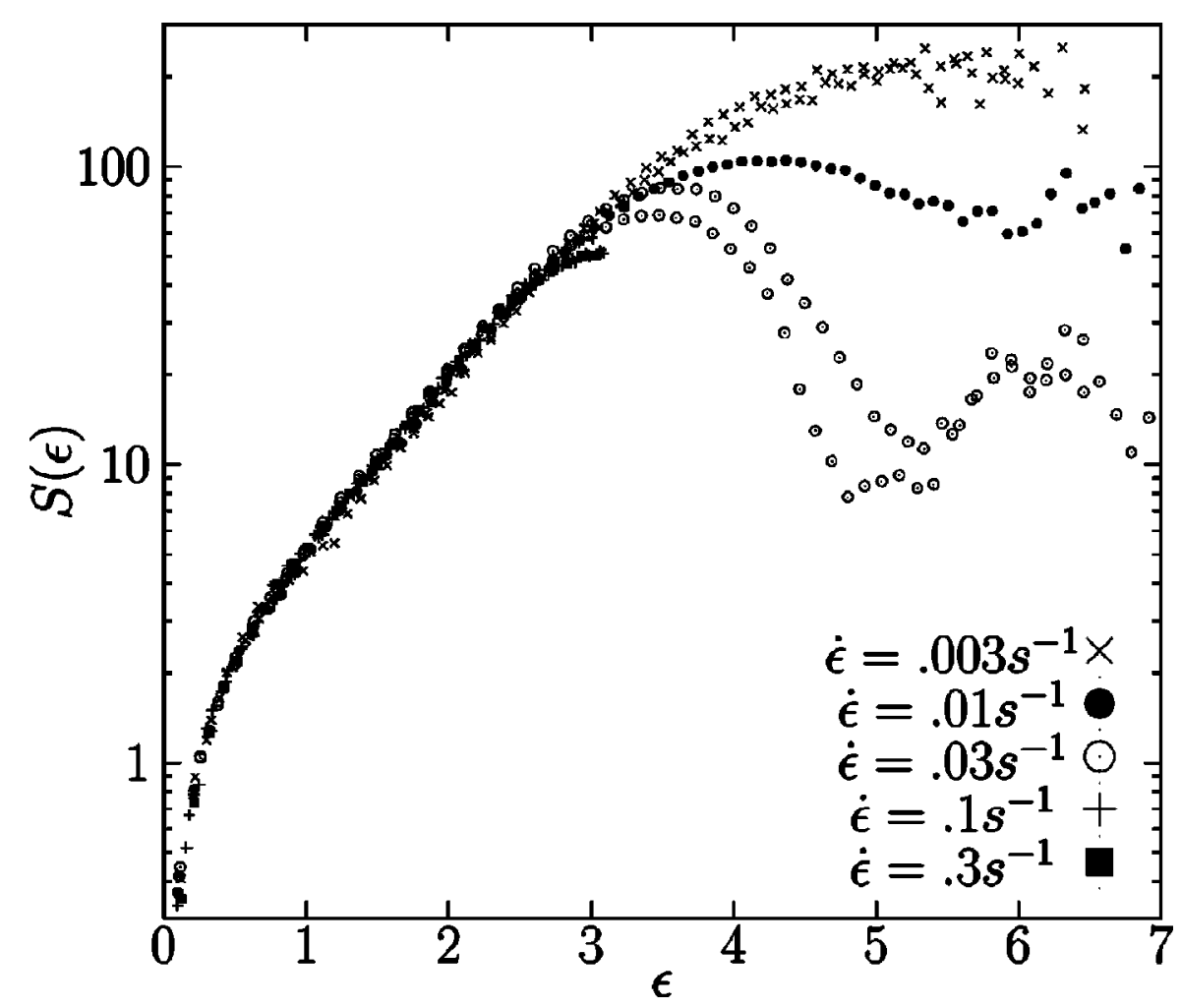

FIG. 8. The strain function, $S(\epsilon)$ defined in Eq. (A1), as a function of the Hencky strain, $\epsilon$. The viscosity measurements, of Lupolen $3020 \mathrm{D}$ measured at $130^{\circ} \mathrm{C}$, are used to calculate $S(\epsilon)$, from Eq. (A2) and from Fig. 1.

\section{References}

Bach, A., K. Almdal, H. K. Rasmussen, and O. Hassager, "Elongational viscosity of narrow molar mass distribution polystyrene," Macromolecules 36, 5174-5179 (2003a).

Bach, A., H. K. Rasmussen, and O. Hassager, "Extensional viscosity for polymer melts measured in the filament stretching rheometer," J. Rheol. 47, 429-441 (2003b).

Bastian, H., "Non-linear viscoelasticity of linear and long-chain-branched polymer melts in shear and extensional flows," Ph.D. thesis, Institut fur Kunststofftechnologie der Universität Stuttgart, 2001.

Gupta, R. K., D. A. Nguyen, and T. Sridhar, "Extensional viscosity of dilute polystyrene solution-Effect of concentration and molecular weight," Phys. Fluids 12, 1296-1318 (2000).

Kolte, M. I., H. K. Rasmussen, and O. Hassager, "Transient filament stretching rheometer II: Numerical simulation," Rheol. Acta 36, 285-302 (1997).

Laun, H. M., and H. Münstedt, "Elongational behaiviour of a low density polyethylene melt. I," Rheol. Acta 17, 415-425 (1978).

McKinley, G. H., and T. Sridhar, "Filament-stretching rheometry of complex fluids," Annu. Rev. Fluid Mech. 32, 375-416 (2002).

McLeish, T. C. B., and R. G. Larson, "Molecular constitutive equations for a class of branched polymers: The pom-pom polymer," J. Rheol. 42, 81-110 (1998).

Münstedt, H., and H. M. Laun, "Elongational behaviour of a low density polyethylene melt. II," Rheol. Acta 18, 492-504 (1979).

Nordmaier, E., U. Lanver, and M. D. Lechner, "The molecular structure of low-density polyethylene 1. Longchain branching and solution properties," Macromolecules 23, 1072-1076 (1990a). 
Nordmaier, E., U. Lanver, and M. D. Lechner, "The molecular structure of low-density polyethylene 2. Particle scattering factors," Macromolecules 23, 1077-1084 (1990b).

Raible, T., A. Demarmels, and J. Meissner, "Stress and recovery maxima in LDPE melt elongation," Polym. Bull. (Berlin) 1, 397-402 (1979).

Rasmussen, H. K., and O. Hassager, "The role of surface tension on the elastic decohesion of polymeric filaments," J. Rheol. 45, 527-537 (2001).

Spiegelberg, S. H., and G. H. McKinley, "The role of end-effects on measurements of extensional viscosity in filament stretching rheometers," J. Non-Newtonian Fluid Mech. 64, 229-267 (1996).

Szabo, P., "Transient filament stretching rheometer part I: Force balance analysis," Rheol. Acta 36, 277-284 (1997).

Szabo, P., and G. H. McKinley, "Filament stretching rheometer: Inertia compensation revisited," Rheol. Acta 42, 269-272 (2003).

Tanner, R. T., and K. Walters, Rheology: An Historical Perspective (Elserier, Amstadam, 1998).

Wagner, M. H., "A constitutive analysis of uniaxial elongational flow data of a low-density polyethylene melt," J. Non-Newtonian Fluid Mech. 4, 3955 (1978).

Wagner, M. H., H. Bastian, P. Hachmann, J. Meissner, H. Münstedt, S. Kurzbeck, and F. Langouche, "The strain hardening behaviour of linear and long-chain-branched polyolefin melts in extensional flows," Rheol. Acta 39, 97-109 (2000).

Wagner, M. H., T. Raible, and J. Meissner, "Tensile stress overshoot in uniaxial extension of a LDPE melt," Rheol. Acta 18, 427-428 (1979).

Wagner, M. H., P. Rubio, and H. Bastian, "The molecular stress function model for polydisperse polymer melts with dissipative convective constraint release,” J. Rheol. 45, 1387-1412 (2001).

Wiest, J. M., “A differential constitutive equation for polymer melts," Rheol. Acta 28, 4-12 (1989). 